JASC 12-2-1

\title{
Analytical Approach of Multicasting-supported Inter-Domain Mobility Management in Sensor-based Fast Proxy Mobile IPv6 Networks
}

\author{
Ha-na Jang ${ }^{\dagger}$, Jong-pil Jeong* \\ ${ }^{\dagger}$ Graduate School of Information and Communications, Sungkyunkwan University, Korea \\ ${ }^{*}$ College of Information and Communication Eng, Sungkyunkwan University, Korea
}

\begin{abstract}
IP-based Wireless Sensor Networks (IP-WSNs) are gaining importance for their broad range of applications in health care, home automation, environmental monitoring, industrial control, vehicle telematics, and agricultural monitoring. In all these applications, a fundamental issue is the mobility in the sensor network, particularly with regards to energy efficiency. Because of the energy inefficiency of network-based mobility management protocols, they can be supported via IP-WSNs. In this paper, we propose a network-based mobility-supported IP-WSN protocol called mSFP, or the mSFP: "Multicasting-supported Inter-Domain Mobility Management Scheme in Sensor-based Fast Proxy Mobile IPv6 Networks". Based on $[8,20]$, we present its network architecture and evaluate its performance by considering the signaling and mobility cost. Our analysis shows that the proposed scheme reduces the signaling cost, total cost, and mobility cost. With respect to the number of IP-WSN nodes, the proposed scheme reduces the signaling cost by $7 \%$ and the total cost by $3 \%$. With respect to the number of hops, the proposed scheme reduces the signaling cost by $6.9 \%$, the total cost by $2.5 \%$, and the mobility cost by $1.5 \%$. With respect to the number of IP-WSN nodes, the proposed scheme reduces the mobility cost by $1.6 \%$.
\end{abstract}

Key words: Wireless Sensor Networks; mSFP; IP-WSN; 6LoWPAN; IEEE 802.15.4

\section{INTRODUCTION}

Recent advancements in micro-electro-mechanical and wireless communication systems have enabled the development of low cost, low power, and multifunctional sensor networks (SNs) that are small in size and can communicate over short distances $[1,2]$. A sensor network is a special type of communication network that is composed of a large number of tiny SNs; these SNs are densely deployed either inside the phenomenon or very close to it [1]. The sensors are generally equipped with data processing and communication capabilities. Accordingly, the sensing circuitry senses the environment surrounding the sensor and transforms that information into an electric signal. It then sends this signal, usually via radio transmitter, to the sink node either directly or through other SNs.

The IPv6 defines the manner in which IPv6 communication is

Manuscript received : Sept. 27, 2012 / revised : Nov. 1, 2012

†Corresponding Author: hnsh77@naver.com

Tel: +82-32-471-8264, Fax: +82-32-471-8264,

Dept. of Information and Communications, Sungkyunkwan

University, Korea to be carried out over the IEEE 802.15.4 interface via the low power wireless personal area network (6LoWPAN) working group of the Internet Engineering Task Force (IETF) [3,4]. Although 6LoWPAN helps to make the wide implementation of IP-WSNs a reality and its end-to-end communication to the external world feasible, excessive tunneling through the air results in increased signaling costs for SNs, making these pursuits difficult. Yet, excessive signaling costs can be limited through the application of IP-WSNs [5]. Moreover, most communication protocols are host-based nowadays, and individual nodes need to participate in mobility related signaling, which is virtually impossible for IP-WSN. Thus, PMIPv6 [9] is a network-based protocol that provides mobility support to any IPv6 host within a restricted and topologically-localized portion of the network, without requiring the host to participate in any mobility-related signaling. 


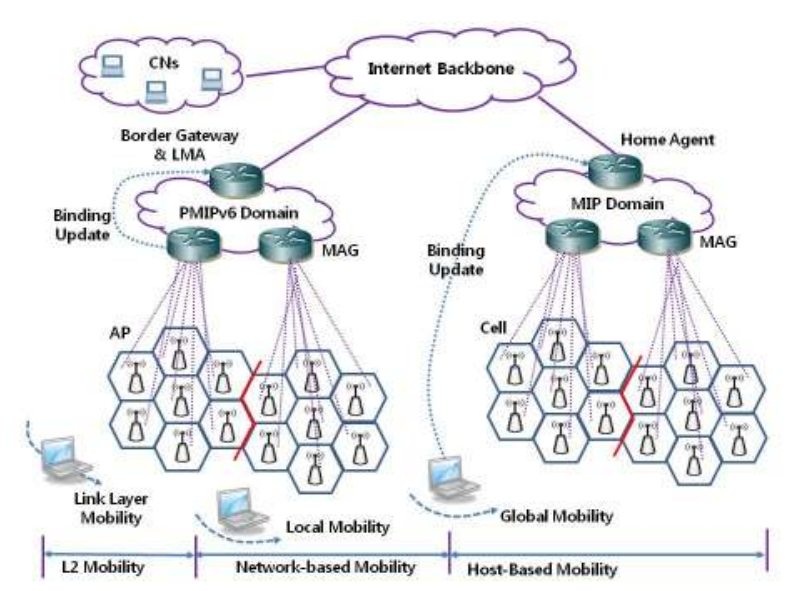

Fig. 1. IP-based mobility management in mobile networks.

6LoWPAN-based IP-WSNs may use the sensor network compatible PMIPv6 to introduce and enhance the mobility scenario in a localized domain. The IP-based mobile network in Figure 1 presents the mobility management scheme. Accordingly, this paper focuses on the mobility in IP-WSNs, highlighting an energy efficient network-based communication protocol for IP-WSNs. In this regard, we propose the mSFP for IP-WSNs, and in our proposed scheme, we use a sensor-based Local Mobility Anchor (LMA), Mobile Access Gateway (MAG), and Sensor Node (SN). We also enable use of the IPv6 header stack. All of these IP devices - in addition to the SNs - make it feasible to implement the mSFP protocol over IP-WSNs.

There are different application areas where IP-WSNs can be used, such as for industrial control, structural monitoring, healthcare, vehicle telematics, and agricultural monitoring [6]. Node-to-node communication is very important since these are the collaborative functions. In these cases, IP-WSNs based on a mesh approach can enhance the communication, where individual SNs can act as routers or fully functional devices. It is discussed in the route over routing issue in 6LoWPAN. Moreover, an mSFP-based IP-WSN facilitates node-to-node seamless communication. Thus, an IP-WSN architecture is proposed for energy efficient mobility of individual SNs or a group of SNs. This paper presents a sensor network-based localized-mobility management protocol in an IP-WSN domain with consideration to the energy efficiency. We propose a mSFP operational architecture, a sequence diagram, and network architecture, and we evaluate the performance of the proposed protocol architecture. Mathematical analysis is conducted to demonstrate the effectiveness of the proposed scheme. Our analytical results show that the proposed scheme effectively reduces the signaling cost and the mobility cost in terms of the number of IP-WSNs and hops compared to PMIPv6 and SPMIPv6 protocols.

The remainder of the paper is organized as follows: Chapter 2 describes the PMIPv6, 6LoWPAN, and multicasting-based mobility management for the related research. The proposed $\mathrm{mSFP}$ protocol architecture and sequence diagram are presented in chapter 3. Chapter 4 presents the performance analysis result. The conclusion of this paper is discussed in chapter 5 .

\section{RELATED WORK}

\section{A. PMIPv6}

The foundation of PMIPv6 is based on MIPv6 in the sense that it extends MIPv6 signaling and reuses many concepts such as the Home Agent (HA) functionality [11,12]. However, PMIPv6 is designed to provide network-based mobility management support to a Mobile Node (MN) in a topologically localized domain [3]. Therefore, an $\mathrm{MN}$ freely participates in any mobility related signaling, and the proxy mobility agent in the serving network performs mobility related signaling on behalf of the MN. Once an MN enters its PMIPv6 domain and performs access authentication, the serving network ensures. The $\mathrm{MN}$ is always on its home network and can obtain its home address on any access network. The serving network assigns a unique home network prefix to each $\mathrm{MN}$, and, conceptually, this prefix always follows the MN. From the perspective of the MN, the entire PMIPv6 domain appears as its home network. Accordingly, it is needless to configure of address at the MN. The new functional entities of PMIPv6 are MAG and LMA [10]. The MAG typically runs on the access router (AR), and its main role is to detect the MN's movements and initiate mobility related signaling with the LMA on behalf of the MN. In addition, the MAG establishes a tunnel with the LMA in order to enable the MN to use an address. It employs a home network prefix and emulates the MN's home network on the access network for each MN. On the other hand, the LMA is similar to the HA in MIPv6. However, it possesses additional capabilities that are required to support the PMIPv6. The main role of the LMA is to maintain reachability to the MN's address while it moves around within a PMIPv6 domain. In addition, the LMA includes a Binding Cache Entry (BCE) for each currently registered $\mathrm{MN}$. The BCE maintained at the LMA is more extended than that of the HA in PMIPv6 with some additional fields, such as the MN identifier, the MN's home network prefix, a flag indicating a proxy registration, and the interface identifier of the bidirectional tunnel between the LMA and MAG. Such information associates an MN with its serving MAG and enables the relationship between the MAG and LMA to be maintained. 


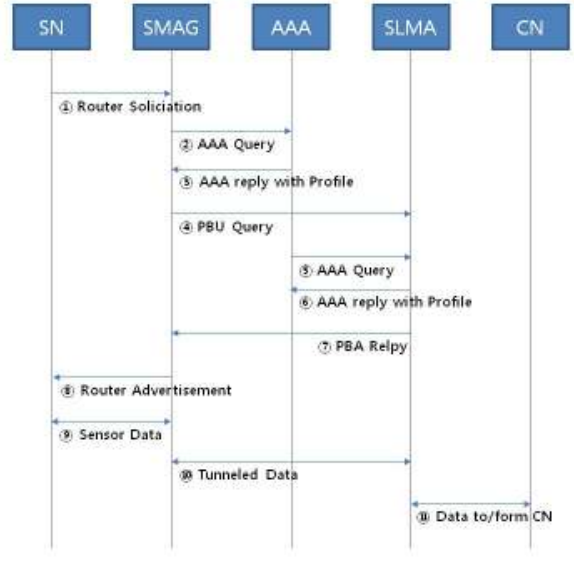

Fig. 2. Message Flow of Sensor-intergrated PMIPv6 Networks.

Figure 2 depicts the sequence diagram of the message flow within the sensor-network-integrated PMIPv6 [9]. 1) When a $\mathrm{SN}$ attaches to a SMAG domain, the access authentication procedure is performed using the SN address. 2) After successful access authentication, the SMAG obtains the SN's profile from the AAA service policy store. 3) The profile contains the SN's address, the SLMA address, the supported address configuration mode, and other associated information. 4) The SMAG sends a Proxy Binding Update (PBU) message, including the MN address, to the SN's SLMA on behalf of the SN. Once the SLMA receives the PBU message, it checks the policy store to ensure that the sender is authorized to send the PBU message. If the sender is a trusted SMAG, the SLMA accepts the PBU message. 5) The SLMA sends a Proxy Binding Acknowledgment (PBA) message, including the MN's home network prefix option, and establishes a route for the SN's home network prefix over the tunnel to the SMAG.

\section{B. 6LOWPAN}

The 6LoWPAN working group of the IERF has defined an adaptation layer for sending IPv6 packets over IEEE 802.15.4. The goal of 6LoWPAN is to reduce the sizes of IPv6 packets to make them fit in 127 byte IEEE 802.15.4 frames. The 6LoWPAN proposal consists of a header compression scheme, a fragmentation scheme, and a method for framing the IPv6 link local address into IEEE 802.15.4 networks [5,6]. The proposal also specifies the enhanced scalabilities and mobility of the sensor networks. The challenge of the 6LoWPAN lies in the sizable differences between an IPv6 network and an IEEE 802.15.4 network. The IPv6 network defines a maximum transmission unit as 1280 bytes, whereas the IEEE 802.15.4 frame size is 127 octets. Therefore, the adaptation layer between the IP layer and the MAC layer must transport IPv6 packets over IEEE 802.15.4 links. The adaptation layer is responsible for fragmentation, reassembly, header compression and decompression, mesh routing, and addressing for packet delivery under the mesh topology. The 6LoWPAN protocol supports a scheme to compress the IPv6 header from 40 bytes to 2 bytes $[7,15]$.

Nevertheless, mobility of 6LoWPAN could give rise to new and exciting applications. One of the possible applications that exploits mobile SNs is healthcare. Patients could wear multiple sensors so that important health parameters - like their heart beat - could be monitored while they are moving. These SNs would form a Wireless Body Sensor Network (WBSN) and use mesh routing in order to transmit their readings to a hub or information control center, which could be a PDA or laptop. Several existing communication technologies have been considered as candidates for the internal and external communication infrastructure of a WBSN. However, most of these protocols have their own shortcomings when they are used in WBSNs. For example, WLAN technology is not suitable for WBSNs as the low-powered WBSN devices have to increase their transmission power in order to avoid interference from other powerful devices like PDAs and notebook computers. Similarly, some communication technologies like Ultra Wideband (UWB) need complex protocols and hardware, which may not be feasible for WSBNs. Previous reports have noted that 6LoWPAN could be one of the most suitable technologies for WBSNs since it is based on the IEEE 802.15.4 specifications [12]. The IEEE 802.15.4 standard suggests a low data rate, low power, less complex protocols and hardware for a SN. Its interaction with IPv6 implies that the SN should easily be interoperable with all other IP networks, including the Internet. This feature, in turn, means that the sensor data can be accessed from anywhere form the world. Alternatively, [13] has proposed the design of a micro-mobility support for SNs, roaming across several Access Points (AP) of a Bluetooth SN. A mechanism to assign IP addresses to both an $\mathrm{AP}$ and a $\mathrm{SN}$ was also proposed so that a $\mathrm{MN}$ would be identified without using the channel number. In addition, they designed a middleware to carry IP packets over the Bluetooth network. However, they assume that the $\mathrm{SN}$ is capable of performing single hop communication even if the $\mathrm{MN}$ is not that close to the AP. Moreover, the MNs are expected to incorporate middleware layer changes in their stack in order to support their mobility. 


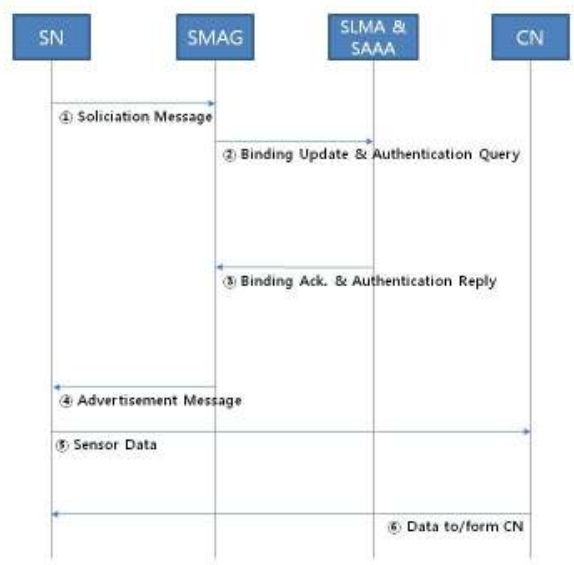

Fig. 3. Message Flow of Sensor-integrated SPMIPv6 Networks.

Figure 3 depicts the sequence diagram of the message flow within a sensor-network-integrated SPMIPv6 [8]. 1) When the location of an IP-SN changes, it sends a solicitation message for the nearest SMAG discovery mechanism. 2) The SMAG then sends a binding update and authentication query to a SLMA integrated with the SAAA. 3) In response to the binding update and authentication query message, the SLMA integrated with the SAAA returns the binding acknowledge and authentication reply message. 4) Finally, the SMAG sends an advertisement message to the respective IP-SN, thereby connecting the IP-SN to the nearest SMAG. 5) The $\mathrm{SN}$ is able to communicate with the corresponding node SPMIPv6. Thus, data can be transmitted from the IP-SN to the correspondence node and vice versa.

\section{Mobility management for multicast support}

A MN does not require specific features, which reflects the concept of network-based mobility management. The Multicast Listener Discovery (MLD) proxy is a function of the supported MLD Membership Report sent from the MN, and is necessary to send the multicast communication to a SN. The specified multicast router function is also necessary because it is a multicast forwarding state management platform for MNs and for multicast data sent from MNs. In some cases, a MLD proxy function is required, depending on the MLD Membership Report. Conceptually, the LMA is connected to the output interface in the multicast routing infrastructure, while the MAG is connected to the input interface at the same time. The MN attached to the MAG receives the multicast data through the established tunnel between the MAG and LMA. The subsequent procedure of the system between the Dredg.PBU message and the RA message is a basic Pmipv6 handover for unicast. The nMAG receives the RS message sent from the $\mathrm{MN}$ and accurately recognizes the connection of the $\mathrm{MN}$, after which the location update on behalf of the MN begins. This process involves the MLD Query, the MLD Membership message, and the Agg.MLD Membership Report, which is sent to the PMIPv6 multicast receiver. The same steps of MLD Query, MLD Membership Report, and Agg.MLD Membership Report are also used for the IPv6 multicast receiver. Since location update, nMAG is part of the action of the multicast router, and it sends the MLD Query message to the MN. The home link of nMAG is emulated because the $\mathrm{MN}$ does not recognize the network movement. Furthermore, the MLD Membership Report message cannot guarantee that he's not requested handover in PMIPv6. It has no meaning. Once the nMAG receives the MLD Membership Report message, it is updated with the MLD Proxy Membership data and the multicast forwarding state. Once the MLD Membership Information has changed, nMAG sends a synthesized Proxy Membership Report message to the MN. Then, once the LMA receives the synthesized MLD Membership Report message, the LMA updates its forwarding state. Thus, the Proxy Mobility Agent in the environment, which is given by PMIPv6, is supported for an IP Mobile MLS. However, the standardization certification for the PMIPv6 MLS in the IEFT MultiMob workgroup needs improvement, and we did not consider the optimization of its specific performance. Our focus was on the development of basic deployment specifications for Mobility-unaware MNs. Because cannot avoid the limit and forcing is applied. First, disconnected services is not been considered of optimization of improve the performance of multicast handover. Accordingly, during the handover, the MN multicast communication is lost. Therefore, Provides continuous handover is not possible. Second, unnecessary multicast communication is the transmission. $\mathrm{MN}$ of last subscribers of multicast service is sends to network during the execution of handover that it is unnecessary multicast communication to previous connected network. These unnecessary transmissions of Multicast communication continue until completion of update of multicast forwarding state for the MN.

\section{MULTICASTING-SUPPORTED INTER-DOMAIN MOBILITY MaNagement SCHEME IN SENSOR-BASED FPMIPV6 (MSFP)}

The mSFP based on the PMIPv6 is proposed to increase the energy efficiency of individual SNs and group SNs. To verify the performance of the proposed scheme, we present a mathematical analysis of the functional structure, sequence diagram, network model, and proposed protocol architecture.

A. System Architecture

Our mSFP is a localized mobility management protocol 
based on PMIPv6, and it consists of a multicasting-based sensor LMA (mSLMA), a multicasting-based sensor MAG (mSMAG), and a SN. Figure 4 depicts the architecture of the proposed $\mathrm{mSFP}$.

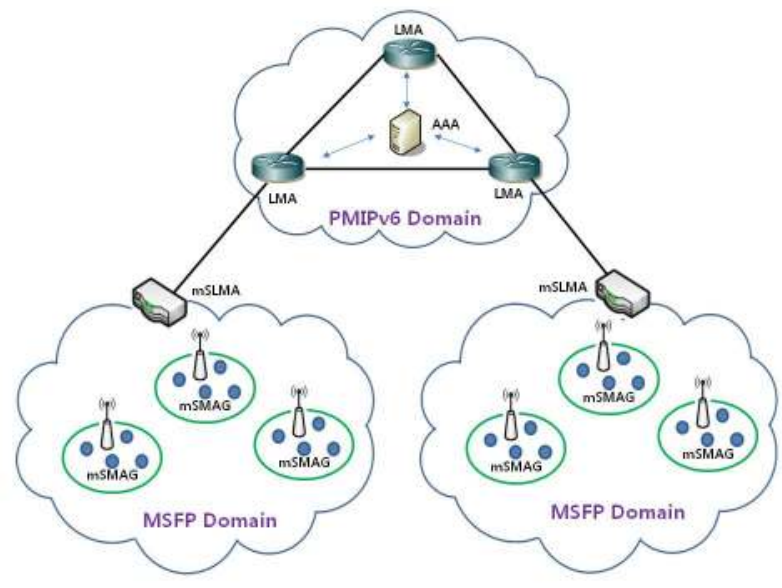

Fig. 4. The network architecture of the proposed mSFP.

The mSLMA acts as a topological anchor point for the entire mSMAG and SN. The main role of the mSLMA is to maintain accessibility to the $\mathrm{SN}$ while the node moves within or outside of the domain. The mSLMA includes a $\mathrm{BCE}$ for each $\mathrm{SN}$, encapsulation and decapsulation functions, and a mSMAG information table. The BCE at the mSLMA is used to hold the information of the mobile SN. The mSLMA has sufficient memory, as well as a sufficient power supply and processing capability. It also acts as the interfacing device between the mSFP and PMIPv6 domains. In this scheme, the Authentication, Authorization, and Accounting (AAA) service has been integrated within the mSLMA (SAAA). The SAAA scheme helps the mSMAG and SN to obtain secured mobility in the MSFP domain and facilitates authentication services for each $\mathrm{SN}$.

The mSMAG acts like a sink node in a sensor network. In the mSFP domain, however, it acts like an access gateway router with a primary function of detecting SN movement and initiating mobility-related signaling with the SN's mSLMA on behalf of the SN. It consists of different functional modules, such as routing, neighbor discovery, adaptation, and interfacing modules, as well as a sensor information table, for the SN and mSLMA. The routing module performs efficient data transmission between individual SNs and facilitates the end-to-end communication. The neighbor discovery module performs neighbor discovery and duplicate address detection functions. The adaptation module performs the task of transmitting the IPv6 packet over the IEEE 802.15.4 link as mentioned with the 6LoWPAN adaptation layer. The sensor information table provides the up-to-date $\mathrm{SN}$ information to the mSLMA. It works closely with the BCE of the mSLMA, and the two interfacing modules communicate with the mSLMA and the SN.

The mSFP domain consists of numerous SNs based on IPv6 addresses and is considered a federated IP sensor domain. Each SN has a tiny TCP/IP communication protocol stack with an adaption layer and an IEEE 802.15.4 interface. This type can forward information to other nodes of similar types, and it can perform information sensing from the environment. In fact, this type of SN acts like a mini sensor router. The other type of SN has the protocol stack and environment sensing capability, but it can forward the sensed information to a nearby mini sensor router node.

\section{B. Operational Architecture}

Figure 5 shows the operational architecture of the mSFP, which includes the mSLMA, mSMAG and SN. Additionally, it shows how these entities communicate with each other through different types of interfaces.

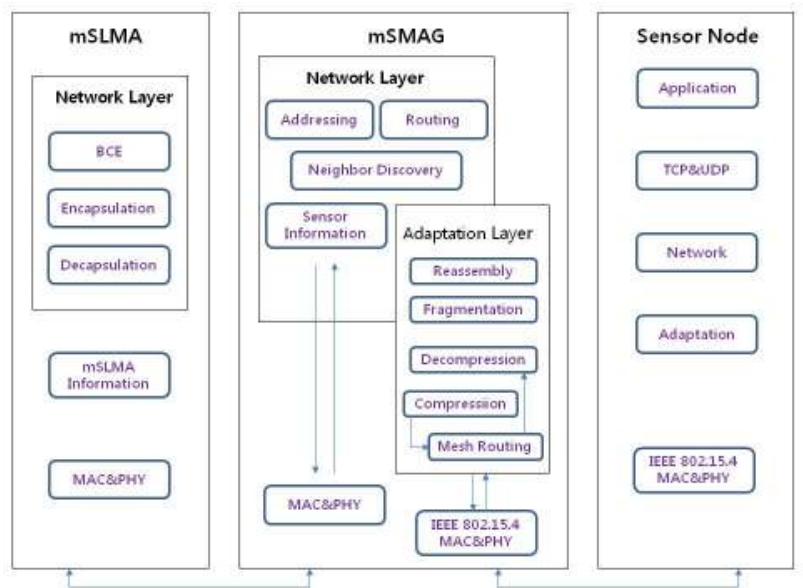

Fig. 5. The operational architecture of the mSFP.

The mSMAG needs two or more interfaces in order to communicate with different access networks, such as the PMIPv6 network. It includes functionality for the network, adaptation, and physical layers. The network layer provides the address, addressing, routing, neighbor discovery mechanisms, as well as the data structure to hold the SN information. The most important layer is the adaptation layer, which ensures that the mesh routing, compression and decompression, and fragmentation and reassembly perform correctly. The physical layer provides access to different physical interfaces. The mSLMA holds network related information such as the BCE, encapsulation, and decapsulation. The mSMAG, SN, and corresponding node (CN) interact in order to make Multicasting Routing possible because it includes the Multicasting Core BCE and 
provides the data structure to hold different information such as new flags, link local addresses for each interface, home prefixes, bi-directional tunnel interface identifiers, access technology, and time stamps. All of the SNs consist of IPv6 addresses for local and global communications.

Figure 6 depicts the sequence diagram of the message flow within the sensor network integration mSFP.

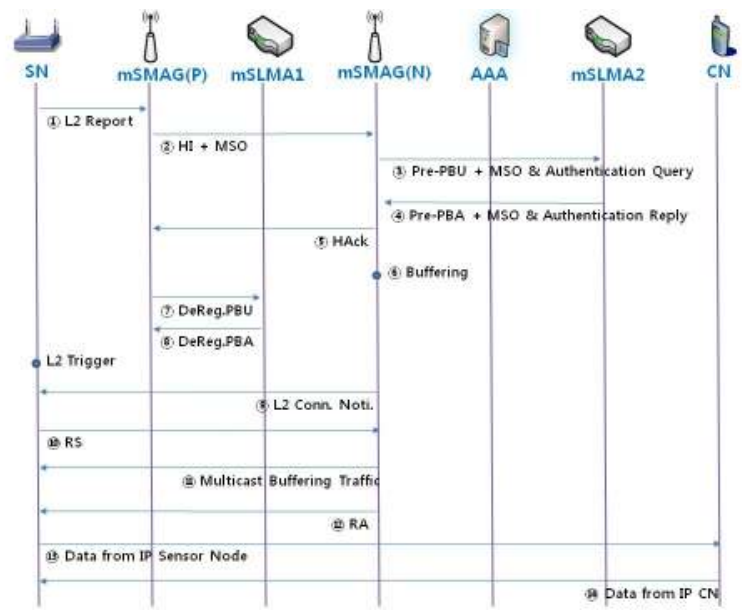

Fig. 6. The multicast-based sequence diagram in PMIPv6.

Step 1: The L2 tigger is passed to the $\operatorname{mMAG}(\mathrm{P})$. The $\mathrm{SN}$ is ready to pass the mSMAG(P) to the mSMAG(N).

Step 2: The mSMAG(P) sends the HI and MSO messages to the mSMAG(N), after which the mSMAG(N) sends the Pre-PBU and MSO Authentication Query to the mSLMA2. In response, the mSLMA2 sends the Pre-PBA and MSO Authentication reply message to the $\operatorname{mSMAG}(\mathrm{N})$, and the mSMAG(N) sends mSMAG(P) a HAck message that includes either an acknowledge (Ack) or a negative acknowledge (NAck).

Step 3: The mSMAG(P) sends the DeRdg.PBU message to the mSLMA1, and the mSLMA1 sends the DeReg.PBA message to the mSMAG(P). Then, the mSMAG(N) informs the information that the $\mathrm{L} 2$ is connected to the $\mathrm{SN}$.

Step 4: Once the SN sends the mSMAG(N) to RS message, mSMAG(N) sends in order to the Multicast Buffering Traffic and RA to SN.

Step 5: Finally, the $\mathrm{SN}$ is communicating with the Corresponding Node $(\mathrm{CN})$ based on the mSFP. Thus, data can be transmitted from the $\mathrm{SN}$ to the $\mathrm{CN}$ and vice versa.

\section{Performance Evaluation}

\section{A. Network Mobility Model}

The mobility of SNs is the major advantage of IP-WSNs over conventional static wireless sensor networks. Mobility is a key concern in the design and performance analysis of
IP-WSNs. The mobility model plays a key role in studying different mobility management strategies such as registration, hand off, and authentication. A mobility model with minimum assumptions that is simple to analyze is very useful for an IP-WSN. Most wireless network performance studies assume that the coverage areas are configured in a hexagonal or square shape (see Figure 7). In this paper, we assume that IP-WSN networks have been configured with a hexagonal topology. Each SN for an IP-WSN area is assumed to have identical movement patterns within and across the IP-WSN. A 2D hexagonal random walk mobility model can then be used to study the movement pattern of the movable SNs. We will use a network model subject in order to modify some of the six-layer personal area network model where $n=6$. In our network model, the IP-WSN consists of a cluster of hexagonal sensor nodes, as shown in Figure 8 [14].

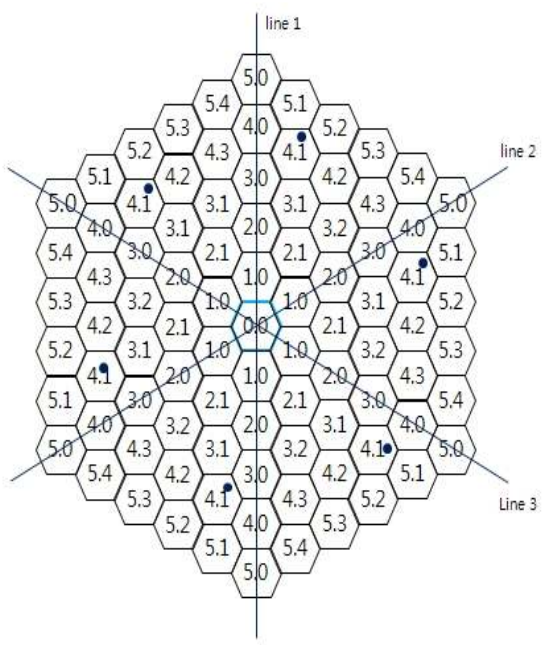

Fig. 7. The six-layer network of the mobility model.

The mSMAG at the center of the IP-WSN area is sublayer 0 . The six-subarea clusters are shown in Figure 7; lines 1-3 divide the cluster into six equal pieces. Exchange of any two pieces has no effect on the structure of the cluster. For example, the cells marked with 4.1 , are at the same relative positions on different pieces; thus, they are grouped together and assigned to the same type [14]. The SNs in cells of the same type will leave the cells with the same routing pattern. A SN can move to any one of its six neighbors with a uniform probability of $1 / 6$. Each sensor node is denoted by $<\mathrm{x}, \mathrm{y}\rangle$, where $\mathrm{x}$ indicates that the mSMAG is in subarea $\mathrm{x}$, and $\mathrm{y}$ is one of the types of subarea $\mathrm{x}$. Sites $\langle 5,0\rangle,\langle 5,1\rangle,\langle 5,3\rangle$, and $<5,4>$ are in the boundary of the IP-WSN and are, therefore, called the boundary states. The state transition diagram of the regular Markov chain corresponding to the random walk model for the six-layer IP-WSN area is shown in Figure 8. Movement into any boundary state indicates inter-IPWSN mobility, which can be used to study binding costs. 


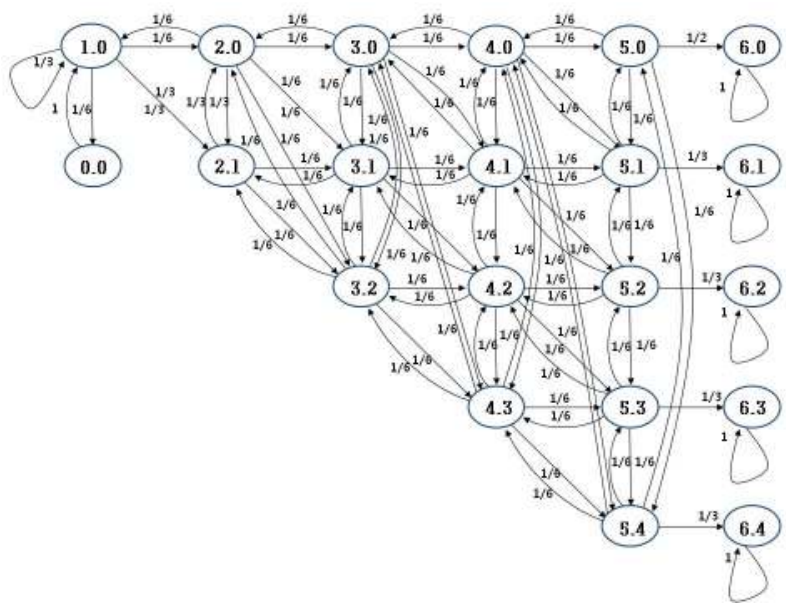

Fig. 8. The state transition diagram of the mobility model.

\section{B. Cost Analysis}

Figure 8 shows the state transition diagram of the mobility model. The properties of the regular Markov chains can be exploited to analyze the behavior of the proposed model [15]. Let $\mathrm{P}$ be the regular transition probability matrix. Then, the steady state probability vector $\pi$ can be solved using the following equations:

$$
\pi P=\pi, \sum_{i=1}^{m} \pi_{i}=1
$$

where $\mathrm{m}$ is the number of states and $\mathrm{P}$ is the fundamental matrix for the regular Markov chain. Then,

$Z=\left[Z_{i j}\right]=(I-P-A)^{-1}$

$A$ is a limiting matrix determined by $P$, and the powers of $P_{n}$ approach the probability matrix A. Each row of A consists of the same probability vector $\pi=\left\{\pi_{1}, \pi_{2}, \pi_{\mathrm{n}}\right\}$, I, e. $A=\{\pi$, where $\}$. When all entries in this column vector equal 1 , it forms the identity matrix, I.

The matrix $\mathrm{Z}$ can be used to study the behavior of the regular Markov chain. Using the matrix involves calculating the number of meanings. For example, $\mathrm{Y}_{\mathrm{j}}(\mathrm{K})$ is the number of times that a process is in state $s_{j}$ in the first $\mathrm{k}$ steps, and $[\mathrm{y}(\mathrm{k})]$ is mean number of times that the process is in state $\mathrm{s}_{\mathrm{j}}$, starting from state $\mathrm{S}_{\mathrm{i}}$.

$M_{i}\left[y_{i}^{(k)}\right] \rightarrow\left(Z_{i j}-\pi_{j}\right)+k \pi_{i}$

The total number of boundary updates in k steps, starting from state $s_{i}$, can be computed from the total number of times that the process is in the boundary states, starting from state $s_{j}$, the initial state. Then, the average number of location updates $\left(\mathrm{U}_{\mathrm{bu}}\right)$ can be given with the following analytical model:

$U_{b u}=M_{i}\left[y_{1}{ }^{(k)}\right]+M_{i}\left[y_{2}{ }^{(k)}\right]+M_{i}\left[y_{3}{ }^{(k)}\right]+M_{i}\left[y_{4}{ }^{(k)}\right]=\sum_{n=1}^{4} M_{i}\left[y_{n}{ }^{(k)}\right]$

We can use the above equation to determine the number of binding update messages. Additionally, because we need to send a binding update message whenever the sensor node moves between IP-WSNs, a binding update message is generated each time a node enters a boundary state. Therefore, we need to determine the expected number of times that the process enters into a boundary state within K steps. Thus, the SNs need to send $\mathrm{U}_{\mathrm{bu}}$ binding update messages, given that the $\mathrm{SN}$ experiences a total of $\mathrm{K}$ transitions between mSMAGs. Accordingly, the ratio of the intra-IP-WSN mobility is denoted as $\mathrm{M}_{\text {intra }}$ and expressed as follows:

$M_{\text {intra }}=\frac{\left(K-U_{b u}\right)}{K}$

Likewise, the ratio of the inter-IP-WSN mobility is denoted as $\mathrm{M}_{\text {inter }}$ and is expressed as

$M_{\text {inter }}=\frac{U_{b u}}{K}$

We have evaluated our proposed model based on signaling cost, mobility cost, and energy consumption. In the subsequent section, we discuss the signaling cost/packet delivery analysis and energy consumption analysis with the help of the different parameters mentioned in Table 1 $[8,9,20]$.

Table I. The System Parameters For Performance Analysis.

\begin{tabular}{|l|l|l|}
\hline Parameter & Description & Value \\
\hline PBU & Proxy Binding Update Message & $48 / 56$ \\
\hline PBA & $\begin{array}{l}\text { Proxy } \\
\text { Acknowledgement Message }\end{array}$ & 4028 \\
\hline $\begin{array}{l}\mathrm{D}_{\text {mSMAG-mSLMA }} \\
\left(\mathrm{D}_{\text {SMAG-SLMA }}\right)\end{array}$ & $\begin{array}{l}\text { Distance between mSMAG and } \\
\text { mSLMA } \\
(\text { Distance between SMAG and } \\
\text { SLMA) }\end{array}$ & 2 \\
\hline $\begin{array}{l}\mathrm{D}_{\text {SN-mSLMA }} \\
\left(\mathrm{D}_{\text {SN-SLMA }}\right)\end{array}$ & $\begin{array}{l}\text { Distance between SN and } \\
\text { mSMAG } \\
\left(\begin{array}{l}\text { Distance between SN and } \\
\text { SMAG) }\end{array}\right.\end{array}$ & 1 \\
\hline$\alpha$ & $\begin{array}{l}\text { Unit transmission cost in a } \\
\text { wireless link }\end{array}$ & 10 \\
\hline$\beta$ & Unit transmission cost in a & 1 \\
\hline
\end{tabular}




\begin{tabular}{|l|l|l|}
\hline Parameter & Description & Value \\
\hline & wired link & \\
\hline RS & Router Solicitation Message & $8 / 16$ \\
\hline RA & Router Advertisement Message & 64 \\
\hline \multirow{2}{*}{$\mathrm{C}_{\mathrm{Sd}}$} & Sensor Mobility Cost & $720 / 80$ \\
& & 0 \\
\hline $\mathrm{C}_{\mathrm{bu}}$ & Binding Update Cost & $0 / 8152$ \\
& & $/ 8168$ \\
\hline$\varepsilon$ & Redirecting Packets to MN & 0.8 \\
\hline$\delta$ & Discarding Packets & 0.2 \\
\hline
\end{tabular}

Mobility cost is evaluated based on signaling cost. To evaluate the total signaling costs, we compare the results of our analytical model with those from MIPv6 and PMIPv6 data.

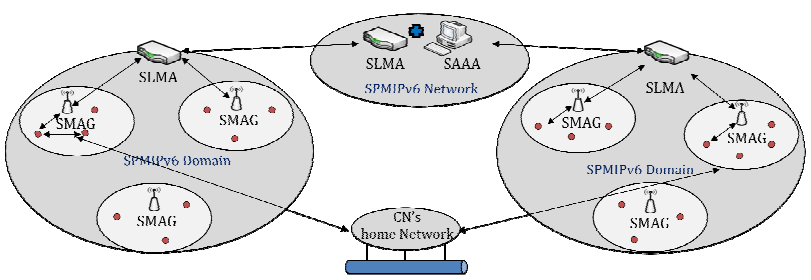

Fig. 9. Network architecture for performance analysis.

Figure 9 depicts the analytical model for the performance analysis of the propose model. It consists of two different mSFP domains that are connected over the PMIPv6-based inter-network. The distance between the mSMAG and SN is denoted by D $_{\text {SN-SMAG }}$ and the distance between the mSMAG and mSLMA is denoted by D $_{\text {SMAG-SLMA }}$. In this analytical model, different distances are used for calculating signaling cost, which is incurred due to the transmission of data and control signal. This cost varies due to different types of signal transmissions.

From equation, the total signaling $\operatorname{cost}\left(\mathrm{TC}_{\mathrm{PMIPv} 6}\right)$ of the proposed scheme based on PMIPv6 can be calculated by summing the signaling $\operatorname{cost}\left(\mathrm{SC}_{\mathrm{PMIPv}}\right)$ and packet delivery $\operatorname{cost}\left(\mathrm{PD}_{\mathrm{PMIPV} 6}\right)$.

$T C_{P M I P V 6}=S C_{P M I I V 6}+P D_{P M I P V 6}$

Note that mSFP is similar to MIPv6, but it reduces the procedure time necessary for MNs. There is no cost of tunneling, so it can be defined as follows: Signaling cost $\left(\mathrm{SC}_{\mathrm{PMIPV} 6}\right)$ is calculated by summing the individual costs of the intra-IP-WSN mobility $\left(\mathrm{M}_{\text {intra }} * \mathrm{C}_{\mathrm{Sd}}\right)$ and the inter-IP-WSN mobility $\left(\mathrm{M}_{\mathrm{inter}} *\left(\mathrm{C}_{\mathrm{Sd}}+\mathrm{C}_{\mathrm{bu}}\right)\right)$, where $\mathrm{C}_{\mathrm{Sd}}$ and $\mathrm{C}_{\mathrm{bu}}$ represent the sensor mobility cost and the binding update cost, respectively.

$$
\begin{aligned}
& S C_{P M I P \vee}=M_{\mathrm{int} r a} C_{s d}^{P M I P \vee 6}+M_{\mathrm{inter}}\left(C_{s d}^{P M I P v 6}+C_{b u}^{P M I P \vee 6}\right) \\
& P D_{P M I P v 6}=\lambda_{p} \cdot t_{L 2} \cdot \eta\left(C_{C N, L M A}+C_{L M A, M A G}+C_{M A G, M N}+P C_{L M A}\right) .
\end{aligned}
$$

Note that $\mathrm{C}_{\mathrm{Sd}}$ and $\mathrm{C}_{\mathrm{bu}}$ are calculated in terms of the PMIPv6.

$C_{s d}^{P M I P v 6}=\alpha \cdot\left(R S_{P M I P v 6}+R A_{P M I P v 6}\right) D_{S N-S M A G}$
$C_{b u}^{P M I P v 6}=\beta \cdot\left(P B U_{P M I P v 6}+P B A_{P M I P v 6}\right) D_{S M A G-S L M A}$

From equation, the total signaling $\operatorname{cost}\left(\mathrm{TC}_{\mathrm{SPMIPV} 6}\right)$ of the proposed scheme based on SPMIPv6 can be calculated by summing the signaling $\operatorname{cost}\left(\mathrm{SC}_{\mathrm{SPMIPv} 6}\right)$ and the packet delivery $\operatorname{cost}\left(\mathrm{PD}_{\mathrm{SPMIPv}}\right)$ such that

$T C_{S P M I P v 6}=S C_{S P M I P v 6}+P D_{S P M I P v 6}$

The process is nearly identical for the SPMIPv6 - i.e.,

$S C_{S P M I P v 6}=M_{\mathrm{intra}} C_{s d}^{S P M I P v 6}+M_{\mathrm{inter}}\left(C_{s d}^{S P M I P v 6}+C_{b u}^{S P M I P v 6}\right)$
$P D_{S P M I P v 6}=\lambda_{p} \cdot t_{L 2} \cdot \eta\left(C_{C N, S L M A}+C_{S L M A, S M A G}+C_{S M A G, S N}+P C_{S L M A}\right) \cdot \varepsilon$

where $\mathrm{C}_{\mathrm{Sd}}$ and $\mathrm{C}_{\mathrm{bu}}$ are calculated in terms of the SPMIPv6.

$C_{s d}^{S P M I P v 6}=\alpha \cdot\left(R S_{S P M I P \vee 6}+R A_{S P M I P v 6}\right) D_{S N-S M A G}$
$C_{b u}^{S P M I P v 6}=\beta \cdot\left(P B U_{S P M I P v 6}+P B A_{S P M I P v 6}\right) D_{S M A G-S L M A}$

Then, from equation, the total signaling $\operatorname{cost}\left(\mathrm{TC}_{\mathrm{mSFP}}\right)$ of the proposed scheme based on mSFP can be calculated by summing the signaling $\operatorname{cost}\left(\mathrm{SC}_{\mathrm{mSFP}}\right)$ and packet delivery $\operatorname{cost}\left(\mathrm{PD}_{\mathrm{mSFP}}\right)$

$T C_{m S F P}=S C_{m S F P}+P D_{m S F P}$

Likewise, for the mSFP,

$$
\begin{aligned}
S C_{m S F P}= & M_{\text {intra }} C_{s d}^{m S F P}+M_{\text {inter }}\left(C_{s d}^{m S F P}+C_{b u}^{m S F P}\right) \\
P D_{m S F P}= & \lambda_{p} \cdot t_{L 2} \cdot \eta\left(C_{C N, m S L M A}+C_{m S L M A, m S M A G(p)}+\right. \\
& \left.C_{m S M A G(p), m S M A G(n)}+C_{m S M A G(n), S N}+P C_{m S L M A}+2 P C_{m S M A G}\right) \cdot \delta .
\end{aligned}
$$

where $\mathrm{C}_{\mathrm{Sd}}$ and $\mathrm{C}_{\mathrm{bu}}$ are calculated in terms of the mSFP.

$C_{s d}^{m S F P}=\alpha \cdot\left(R S_{m S F P}+R A_{m S F P}\right) D_{S N-m S M A G}$

$C_{b u}^{m S F P}=\beta \cdot\left(P B U_{m S F P}+P B A_{m S F P}\right) D_{m S M A G-m S L M A}$

Note that $\mathrm{C}_{\mathrm{bu}}^{\mathrm{mSFP}}$ is the value of the proposed network architecture based on multicasting to perform the binding 
update and does not occur because of cost.

\section{Numerical Results}

In this section, we present the results of experiments evaluating the performance of our scheme, and we compare the performance of our proposed scheme to the PMIPv6, SPMIPv6, and mSFP. We fix the signaling cost and mobility related cost for the number of IP-based SNs and number of hops in order to evaluate the consequences of our proposed scheme with these other protocols and to summarize the key characteristics of our proposed approach as compared to the approach of the PMIPv6, SPMIPv6, and mSFP. We implemented the model and evaluated the parameters, such as the signaling cost and mobility cost, as presented in this paper.

Figure 10 depicts the signaling cost with respect to the number of IP-WSN nodes for the PMIPv6, SPMIPv6, and mSFP. Signaling cost increases as the number of IP-WSN nodes increases. However, the mSFP incurs a much lower signaling cost compared to the PMIPv6 and SPMIPv6 for increasing numbers of IP-WSN nodes. With respect to the number of IP-WSN nodes, the proposed scheme reduces the signaling cost by $7 \%$, as compared to the PMIPv6 and SPMIPv6.

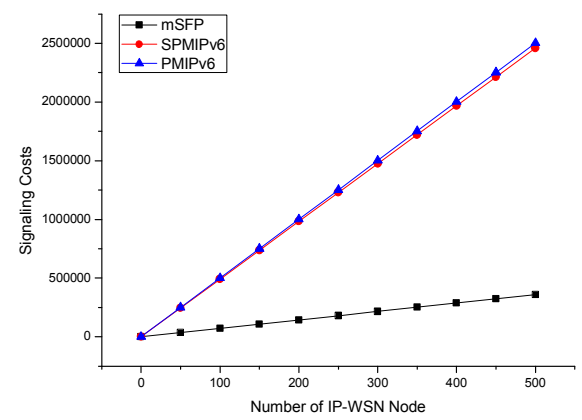

Fig. 10. Signaling Cost as a function of the number of IP-WSN nodes

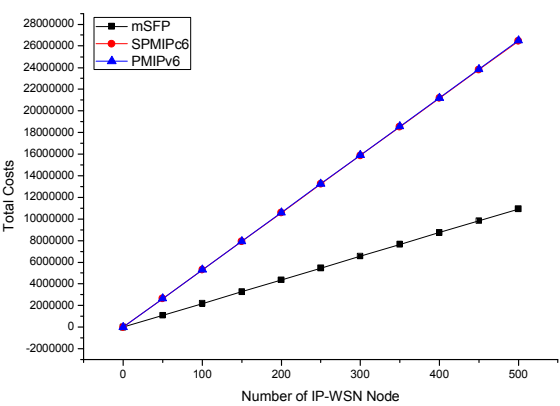

Fig. 11. Total Cost as a function of the number of IP-WSN nodes.
Figure 11 depicts the total cost with respect to the number of IP-WSN nodes in terms of the PMIPv6, SPMIPv6, and mSFP. The total cost increases as the number of IP-WSN nodes increases. However, mSFP incurs a much lower total cost compared to the PMIPv6 and SPMIPv6 for increasing numbers of IP-WSN nodes. With respect to the number of IP-WSN nodes, the proposed scheme reduces the total cost by 3\%, as compared to PMIPv6 and SPMIPv6.

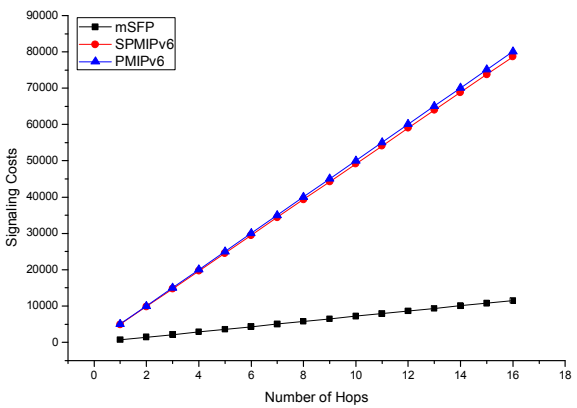

Fig. 12. Signaling Cost vs. Number of Hops.

Figure 12 depicts the signaling cost with respect to the number of hops. In this case, we considered a maximum 15 hops, and we found that the signaling cost increases linearly as the number of hops increases. The proposed scheme shows better performance with respect to both the PMIPv6 and SPMIPv6, and the signaling cost increases in a linear pattern. With respect to the number of hops, the proposed scheme reduces the signaling cost by $6.9 \%$, as compared to the PMIPv6 and SPMIPv6.

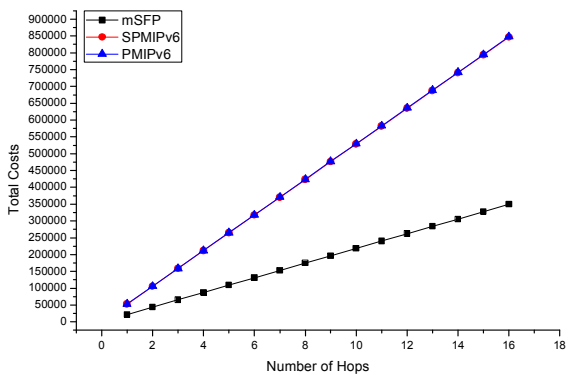

Fig. 13. Total Cost vs. Number of Hops.

Figure 13 depicts the total signaling cost with respect to the number of hops. In this case, we considered a maximum 15 hops, and we found that the signaling cost increases linearly as the number of hops increases. The proposed scheme shows better performance with respect to both the PMIPv6 and SPMIPv6, and the total cost increases in a linear pattern. With respect to the number of hops, the proposed scheme reduces the signaling cost by $2.5 \%$, as compared to PMIPv6 and SPMIPv6. 


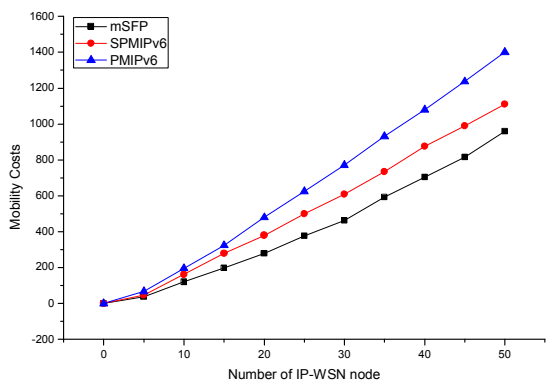

Fig. 14. Mobility Cost vs. Number of IP-WSN nodes.

Figure 14 depicts the mobility cost with respect to the number of IP-WSN nodes in terms of the PMIPv6, SPMIPv6, and mSFP. The mobility cost increases as the number of IP-WSN nodes increases. With respect to the number of IP-WSN nodes, the proposed scheme reduces the mobility cost by $1.6 \%$, as compared to the PMIPv6 and SPMIPv6.

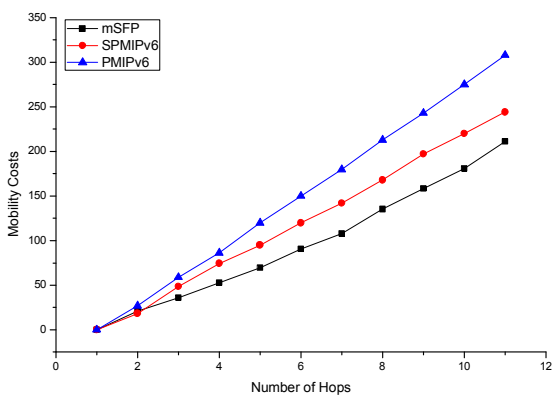

Fig. 15. Number of Hops vs. Mobility Cost.

Figure 15 depicts the mobility cost with respect to the number of hops in terms of the PMIPv6, SPMIPv6, and mSFP. The mobility cost increases as the number of IP-WSN nodes increases. With respect to the number of hops, the proposed scheme reduces the mobility cost by $1.5 \%$, as compared to the PMIPv6 and SPMIPv6.

\section{Conclusion}

The mobility in IP-WSN environments is an important issue that should be evaluated for energy efficiency. In this paper, we proposed a network-based mobility-supported IP-WSN protocol called the "Multicasting-based Inter-Domain Mobility Management Scheme in Sensor-based Fast Proxy Mobile Ipv6 Networks (mSFP) ". We evaluated its performance by analyzing the signaling and mobility costs. Our proposed network architecture does not occur the binding update cost. Therefore, analytical results show that our scheme (mSFP) effectively reduces the signaling cost, total cost, and mobility cost compared to the PMIPv6 and SPMIPv6 protocols.

\section{Acknowledgment}

This research was supported by Basic Science Research Program through the National Research Foundation of Korea(NRF) funded by the Ministry of Education, Science and Technology(2011-0027030). Corresponding author: Jongpil Jeong.

\section{References}

[1] lan F, Akyildiz, and Weilian Su, "A survey on sensor networks," IEEE communication Magazine, Vol. 40, pp. 102-114, August 2002.

[2] Kemal Akkaya, Mohamed Tounis, "A survey on routing protocols for wireless sensor networks," Ad hoc Network, Vol. 3, pp.325-349, March 2005.

[3] Montenegro G., Kushalnagar N, and Hui J W, "Transmission of IPv6 Packets over IEEE 802.15.4 Networks," IETF RFC 4944, September 2007.

[4] Kushalnagar N, Montenegro G, and Schumacher C, "IPv6 over Low-Power Wireless Personal Area Networks (6LoWPANs)," IETF RFC 4919, August 2007.

[5] Zach, S.; Carsten, B. 6LoWPAN: The wireless Embeded Internet, David Hutchios, Serge Fdida, Joe Sventek; John Wiley \& Sons Ltd: Chichester, West Sussex, UK, 2009.

[6] Kim E, Kaspar D, and Chevrollier N, "Design and Application Spaces for 6LoWPANs," IETF internet-Draft, July 2009.

[7] Kong K S, Lee W, and Han Y H, "Mobility Management for Ali-IP mobile networks: mobile IPv6 vs. Proxy mobile IPv6," IEEE Wirel Commun, Vol. 15, PP. 36-45, July 2008

[8] Motaharul Islam, Eui Nam Huh, "Sensor Proxy Mobile(SPMIPv6)-A Novel Scheme for Mobility Supported IP-WSNs," Sensor, PP.1865-1887, November 2011.

[9] Gundavelli S, Leung K, Devarapalli V, Chowdhury K, and Patil B, "Proxy Mobile IPv6," IETF RFC 5213, August 2008.

[10] Chalmers R.C, Almeroth K C, "A mobility gateway for small-device networks," In Proceedings of Second IEEE Annual Conference on Pervasive Computing and Communications Washington DC USA, June 2004.

[11] Kim J H, Hong C S, and Taeshik S, "A Lightweight NEMO protocol to support 6LoWPAN," ETRI Journal, Vol. 30, pp. 685-695, October 2008

[12] Istepanian R, Jovanov E, and Zhang Y, "Guest Editorial Introduction to the Special Section on M-Health: Beyond Seamless Mobility and Global Wireless Health-care connectivity," IEEE Transactions on Information Technology In Biomedicine," Vol. 8, pp.405-414, 2004.

[13] Ni X, Shi W, and Ni, "Design of Micro Mobility Support in Bluetooth Sensor Networks," In IEEE International Conference on Industrial Informatics , pp. 150-154, August 2006.

[14] Akyildiz I F, Lin Y B and Lai W R, "A new random walk model for PCS networks," IEEE Journal on selected areas in communications, Vol. 18, pp. 1254-1259, July 2000.

[15] Ching K H, Shenoy N A, "2D Random walk mobility model for location management studies in wireless network" IEEE Transactions On vehicular Technology, Vol. 53, pp. 413-424, March 2004.

[16] Heinzelman W R, Chandrakasna A, and Balakrishnan H, "Energy-Efficient Communication Protocol for Wireless 
Micro sensor Networks," In Proceedings of the 33rd Annual Hawaii international Conference on System Sciences, Vol.8, pp.8020-8030, January .2000.

[17] Pathan A S K, Hong C S, "SERP: secure energy-efficient routing protocol for densely deployed wireless sensor networks," Ann. Telecommun, Vol. 63, pp.529-541, July 2008.

[18] Razzaque M A, Hong C S, "Analysis of energy-tax for multipath routing in wireless sensor networks," Ann. Telecommun. Vol. 65, pp. 117-127, May 2009.

[19] Dhanajay S, Lee H J, Chung W Y, "An Energy Consumption Technique for Global Healthcare Monitoring Applications," In Proceedings of International Conference on Information Sciences, pp. 539-542, November 2009.

[20] Md. Motaharul Islam and Eui-Nam Huh, "A Novel addressing Scheme for PMIPV6 Based Global IP-WSNs," Sensors, PP.8430-8455, November 2011.

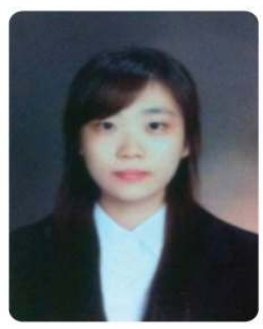

Hana Jang graduated from Yuhan College in 2011 and received her Information \& Communication Engineering degree, with honors, from Yuhan College. Now, She is student at graduate school of SungkyunKwan university.

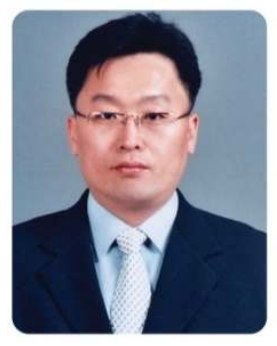

Jongpil Jeong received his B.S. degree in engineering from Sungkyunkwan University and the M.S. and Ph.D. degrees in computer engineering from Sungkyunkwan University, Suwon, Korea, in 2003 and 2008, respectively.

He was a Research Professor with Sungkyunkwan University in 2008-2009 and 2011, and a visiting professor with the Department of Interaction Science in Sungkyunkwan University in 2009-2010. He started his academic profession at Sungkyunkwan Univeristy, Korea in 2012 as an assistant professor. His research interests include mobile computing, mobility management for vehicular networks, sensor networking, protocol operation based performance analysis, Internet security, MIPv6 and ubiquitous computing. 